\title{
TUTORIAL ON OPTIMISATION VIA SIMULATION: HOW TO CHOOSE THE BEST SET UP FOR A SYSTEM
}

\author{
Dr Christine Currie \\ University of Southampton \\ Highfield Road \\ Southampton \\ SO17 1BJ \\ christine.currie@soton.ac.uk
}

\author{
Dr. Thomas Monks \\ University of Exeter Medical School \\ Heavitree Road \\ Exeter \\ EX1 1TE \\ t.m.w.monks@exeter.ac.uk
}

\begin{abstract}
In this tutorial we consider the problem of finding the best set up to use for a system, where the objective is measured using the output of a stochastic simulation model. What makes this a difficult problem is that the output is stochastic and consequently changes in each replication. Optimisation via simulation is a vast topic and we restrict ourselves to a small part of it - ranking and selection - in which a small number of discrete options are being compared. We describe two of the best-used methods, $\mathrm{KN}++$ and OCBA. In these algorithms, just one solution is returned at the end of the optimisation and there is a single objective. We also discuss variations including best subset selection, multi-objective optimisation via simulation, and the minimisation of the expected opportunity cost. The tutorial is accompanied by a Github repository which includes Python code for the algorithms we describe here.
\end{abstract}

\section{Keywords:}

Optimisation via simulation, simulation, ranking and selection

\section{INTRODUCTION}

Optimisation via simulation (OvS) methods use the simulation as a proxy for the real system and the OvS algorithms will choose how to experiment on the simulation model in order to identify the optimal solution as efficiently as possible. Using mathematical notation, we wish to minimise an output $f(\mathbf{x})$, where $\mathbf{x}$ is a vector of decision variables and $f(\mathbf{x})$ is the expected value of the random output $Y(\mathbf{x})$,

$$
f(\mathbf{x})=\mathbb{E}[Y(\mathbf{x})]
$$

We assume that the output $f(\mathbf{x})$ is a single number (or scalar) in most of what follows but in a multi-objective problem, it becomes a vector.

Hong and Nelson (Hong and Nelson 2009) provide a useful classification of simulation optimisation problems, dividing them into three main groups.

1. The feasible region for $\mathbf{x}$ has a small number of discrete solutions, e.g. deciding between several different set-ups for a hospital to maximize throughput.

2. The vector of decision variables $\mathbf{x}$ is continuous, e.g. choosing the intervention or mix of interventions that leads to the least deaths when treating an infectious disease

3. The vector of decision variables $\mathbf{x}$ is discrete and integer ordered, e.g. optimizing the number of call centre staff on duty to minimize costs subject to constraints on response times. 


\section{Currie and Monks}

We would recommend The Handbook of Simulation Optimisation edited by Michael $\mathrm{Fu}(\mathrm{Fu} 2015)$ as a good place to find out more about appropriate algorithms for these three different classes of problem along with tutorial papers from the Winter Simulation Conference.

In this tutorial we will focus on the first group of problems and the algorithms used to solve them, typically referred to as ranking and selection algorithms. We discuss the characteristics of ranking and selection problems in the next section and some variations on the basic single-objective, single-optimum setting. During the tutorial, we will make use of examples to illustrate the algorithms we are introducing here and we refer the reader to the Github repository for more details of these (https://github.com/TomMonks/ovs-tutorial).

Two main algorithms exist within the literature for solving these ranking and selection problems: indifference zone procedures such as $\mathrm{KN}++$ and optimal computing budget allocation or OCBA methods. We describe each of these in turn in Sections 3 and 4. There are numerous extensions to these algorithms and also avenues for future research, which we touch on relatively briefly in Section 5 before concluding and summarising the key messages.

Before continuing it is worth defining a few key terms that we will use throughout the tutorial.

- Probability of Correct Selection (PCS): the probability that the option(s) output by the OvS algorithm is the true minimum. For a real problem, this must be estimated based on the simulation output but when testing algorithms on testbeds with known solutions, this is measured as the proportion of times that the algorithm finds the correct result.

- Expected Opportunity Cost (EOC): the estimated cost associated with choosing the wrong option. This can be a more practical objective to use as it distinguishes between cases where the cost difference between neighbouring options is small/large.

Both of the examples we use here and all of the algorithms that we introduce are included in a Github repository at https://github.com/TomMonks/ovs-tutorial and are free to access and download.

\section{RANKING AND SELECTION}

Ranking and selection algorithms are used when the number of options available is small, i.e. $\mathbf{x}$ can take only a few discrete values, and it is possible to sample at each of these values. The difficulty lies in the fact that the output of the simulation model is stochastic and that we have only a limited time or computational budget for experiments.

Assume that we are comparing $m$ different options for the system set up, $i=1, \ldots, m$. Each of these options has a true mean $\mu_{i}$ that is unknown to the experimenter and we wish to return the system that has the smallest $\mu_{i}$. We obtain estimates of the $\mu_{i}$ by running the simulation model $n_{i}$ times for each option $i$ and finding the sample averages of the output variables $\mathbb{E}\left[Y\left(\mathbf{x}_{i}\right)\right]$. Ranking and selection algorithms aim to choose the $n_{i}$ in such a way that the computation is as efficient as possible.

The original algorithms for solving these problems used two stages, e.g., see (Koenig and Law 1985) and (Chick and Inoue 2001), where in the first stage, each of the options is tested with the simulation model and in the second stage the number of subsequent observations of each system is optimised to ensure that either the PCS is guaranteed or, where the computational budget is fixed, the PCS is maximised. Two-stage approaches have the benefit that there is relatively little communication between the simulation model and the software running the ranking and selection and this can be beneficial when this communication is time-consuming. A further benefit of two-stage procedures is that they can be easier to program and use for non-experts in optimization, as discussed in (Monks and Currie 2018).

The majority of recent work in ranking and selection uses sequential algorithms in which the results of a simulation replication are fed back into the optimisation algorithm after each replication (or a small number of replications) to allow it to choose which option to test next. This allows the algorithms to react quickly to the output of the simulation model and hence improves their efficiency over the two-stage models. It is these algorithms that we focus on in this tutorial, considering first the $\mathrm{KN}++$ algorithm introduced by Kim 


\section{Currie and Monks}

and Nelson (Kim and Nelson 2006) and second the OCBA algorithm introduced by Chen and described in some detail in (Chen and Lee 2010).

There are many variations of the most basic single objective, single result problem and we do not have space to consider them all here. One which is particularly useful when helping with complex decisions is the best subset selection problem, in which the algorithm returns the top $m$ options rather than just the top one. This allows a decision-maker to choose between several "good" solutions, allowing them to take into account factors that cannot be incorporated into the simulation model. In Section 4.1 we describe a sequential algorithm that can be used to solve this problem.

(Branke et al. 2007) compares different ranking and selection algorithms. They consider both OCBA and $\mathrm{KN}++$, as we do here, but also include a value of information procedure or VIP. A VIP will allocate samples in such a way to maximize the expected value of information to be obtained from them. In the interests of space, we do not include these algorithms in this tutorial and would advise an interested reader to search for relevant references in (Branke et al. 2007).

\section{INDIFFERENCE ZONE PROCEDURES}

Indifference zone procedures provide a guarantee of finding the best system with a high probability $(1-\alpha)$ when the long-run average of the best system is at least $\delta$ better than that of the second-best system. Both $\alpha$ and $\delta$ are set by the decision-maker, with $\alpha$ typically being set to 0.05 or 0.10 to provide $95 \%$ or $90 \%$ confidence intervals respectively but it must be set such that $1-\alpha>1 / k$, where $k$ is the number of systems. The indifference zone is defined by $\delta$, the smallest difference in mean that would be significant to a decision maker.

We describe a sequential indifference zone procedure here, $\mathrm{KN}++$, named after Kim and Nelson who describe their algorithm in (Kim and Nelson 2006). This extends the original $K N$ algorithm, updating the variance estimator as more data are obtained, and is the standard indifference zone procedure, known for its efficiency but also its PCS guarantee. The procedure begins by running simulations for all systems and, as it proceeds, it will eliminate systems where the difference in their means and that of the best system exceeds some threshold, as described in the algorithm below. The procedure ends when only one system remains.

\section{$\mathrm{KN}++$ Procedure}

1. Specify $\alpha, \delta$ as discussed above; $n_{0}>2$, the number of replications of each system run during the initialisation step; $\eta$, the number of replications to make for each of the remaining systems at each step of the procedure.

2. Define $I$ as the set of non-eliminated systems, $I \leftarrow\{1, \ldots, k\}$ and set the number of replications $n \leftarrow 0$. Set $\tau \leftarrow n_{0}$.

3. While $|I|>1$

(a) Run $\tau$ replications of each system in $I$. Set $n \leftarrow n+\tau$. Set $\tau=1$.

(b) Update the sample means $\bar{x}_{i}$ and sample variances $\sigma_{i}^{2}$ for each $i$ in $I$. Set

$$
\eta \leftarrow 0.5\left\{\left[2\left(1-(1-\alpha)^{1 /(k-1)}\right)\right]^{-2 /(n-1)}-1\right\}
$$

and $h^{2} \leftarrow 2 \eta(n-1)$.

(c) Find the difference in the means of all pairs of systems in $I, d_{i j} \leftarrow \bar{x}_{i}-\bar{x}_{j}$ for all $i, j \in I$ and $i>j$. Set $\varepsilon_{i j}=\max \left\{0, \delta / 2 n\left(h^{2}\left(\hat{\sigma}_{i}^{2}+\hat{\sigma}_{j}^{2}\right) / \delta^{* 2}-n\right)\right\}$. If $d_{i j}>\varepsilon_{i j}$ then remove $i$ from $I, I \leftarrow \backslash I\{i\}$ else if $d_{i j}<-\varepsilon_{i j}$ then remove $j$ from $I, I \leftarrow I \backslash\{j\}$.

4. Return remaining system as the best. 


\section{Currie and Monks}

\section{OCBA}

The basic idea of OCBA procedures is that additional replications are allocated to systems so as to best optimise the PCS. Effectively this means that the majority of the computational effort should be focused on competitive systems and little effort should be allocated to systems unlikely to be the best. This leaves us with a Catch 22 situation in that without carrying out all of the additional replications we cannot say exactly how the sampling will improve the PCS estimate and which systems are most important to simulate. However, it is possible to estimate the improvement and estimating the improvement in PCS from making additional samples with each system is the basis of determining how replications should be allocated. We refer readers to the excellent book by Chung Hun Cheng and Loo Hay Lee (Chen and Lee 2010) for the full technical details of OCBA and provide only the algorithm in what follows.

\section{OCBA Procedure}

1. Specify $n_{0} \geq 2$, the number of replications to make of each system during initialisation; $k$, the number of systems/options being compared; $\eta$ the number of replications to make at each stage; $T$, the total number of replications. For ease, set $T-k n_{0}$ to be a multiple of $\eta$.

2. Run $n_{0}$ replications for each design, set $l \leftarrow 0$ and $N_{i}^{l} \leftarrow n_{0}, i=1, \ldots, k$.

3. Loop while $\sum_{i=1}^{k} N_{i}^{l} \leq T$.

(a) Update the relevant statistics: sample mean, $\bar{X}_{i}=\frac{1}{N_{i}^{l}} \sum_{j=1}^{N_{i}^{l}} X_{i j}$; sample standard deviation, $s_{i}=\sqrt{\sum_{j=1}^{N_{i}^{l}}\left(X_{i j}-\bar{X}_{i}\right)^{2} /\left(N_{i}^{l}-1\right)} ; b=\arg \min _{i} \bar{X}_{i}$.

(b) Allocate the new budget of $\eta$ replications so that $\frac{N_{i}^{l+1}}{N_{j}^{l+1}}=\left(\frac{s_{i}\left(\bar{X}_{b}-\bar{X}_{j}\right)}{s_{j}\left(\bar{X}_{b}-\bar{X}_{i}\right)}\right)^{2}$ for all $i \neq j \neq b$ and $N_{b}^{l+1}=s_{b} \sqrt{\sum_{i=1, i \neq b}^{k}\left(\frac{N_{i}^{l+1}}{s_{i}}\right)^{2}}$

(c) Perform an additional $\max \left(N_{i}^{l+1}-N_{i}^{l}, 0\right)$ replications for design $i, i=1, \ldots, k ; l \leftarrow l+1$.

\subsection{OCBA-M}

The OCBA-M algorithm was introduced in (Chen et al. 2008) and has the objective of finding all of the top $m$ designs for a simulated system. There are situations where a decision maker wishes to choose between a set of good designs, particularly where there are factors that cannot be included in a simulation model (e.g. political considerations). Top- $m$ designs can also be beneficial as part of a global optimization routine where they are used to generate an elite set of solutions for optimisation in a subsequent stage.

\section{OCBA- $m$ Procedure}

1. Specify $n_{0} \geq 5$, the number of replications to make of each system during initialisation; $k$ the total number of systems; $m ; \eta$, the number of replications to make at each stage; $T$, the maximum number of replications where $T-n_{0}$ is a multiple of $\eta ; m$ the number of designs to include in the subset.

2. Initialise by running $n_{0}$ replications for each design, setting $l \leftarrow 0$ and $N_{1}^{l}, N_{2}^{l}, \ldots, N_{k}^{l}=n_{0}$.

3. Loop while $\sum_{i=1}^{k} N_{i}^{l} \leq T$.

(a) Update statistics as follows. Calculate sample means for each system, $\bar{X}_{i}=\frac{1}{N_{i}^{l}} \sum_{j=1}^{N_{i}^{l}} X_{i j}$ and sample standard deviations $s_{i}=\sqrt{\sum_{j=1}^{N_{i}^{l}}\left(X_{i j}-\bar{X}_{i}\right)^{2} /\left(N_{i}^{l}-1\right)}$, and compute $\hat{\sigma}_{i}=s_{i} / \sqrt{N_{i}^{l}}$ and $c=\left(\hat{\sigma}_{i_{m+1}} \bar{X}_{i_{m}}+\hat{\sigma}_{i_{m+1}} \bar{X}_{i_{m+1}}\right) /\left(\hat{\sigma}_{i_{m}}+\hat{\sigma}_{i_{m+1}}\right)$; update $\delta_{i}=\bar{X}_{i}-c$ for $i=1, \ldots, k$.

(b) Allocate computer budget as follows. The new computing budget increases by $\eta$ and new replications are allocated so that $\frac{N_{1}^{l+1}}{\left(s_{1} / \delta_{1}\right)^{2}}=\frac{N_{2}^{l+1}}{\left(s_{2} / \delta_{2}\right)^{2}}=\ldots=\frac{N_{k}^{l+1}}{\left(s_{k} / \delta_{k}\right)^{2}}$. In practice, this can be achieved by setting $N_{i}^{l+1} \leftarrow N_{i}^{l}, i,=1, \ldots, k$ and ordering the systems in non-decreasing order 


\section{Currie and Monks}

of $\frac{N_{i}^{l+1}}{\left(s_{i} / \delta_{i}\right)^{2}}$. Until $\eta$ observations have been allocated in this stage, add one to the system at the top of the list and reorder.

(c) Simulate an additional $\max \left(N_{i}^{l+1}-N_{i}^{l}, 0\right)$ for design $i, i=1, \ldots, k ; l \leftarrow l+1$.

\section{WHAT NEXT?}

Many problems that we consider in the OvS area are multi-objective and there are several ways of dealing with this. The review paper by Susan Hunter and co-authors (Hunter et al. 2019) provides an excellent overview of such multi-objective simulation optimisation problems and is designed as an advanced tutorial. They have a wider remit than just ranking and selection problems, considering problems with both integerordered decision variables and continuous decision variables. A concept used extensively in multi-objective optimisation is that of a Pareto set. We refer readers to (Hunter et al. 2019) for a formal definition and describe it here as a set of solutions for which one of the objective values is strictly better than any other that can be obtained feasibly, and no improvement can be made on any of the other objective functions by moving to a different feasible point. Identifying the Pareto set can then form the first part of a methodology in which the decision-maker either chooses from the full Pareto set, or obtains additional information from a decision-maker at interim points during the optimisation to guide it to the preferred solution.

Recent work in the area reflects the growing use of cloud computing in simulation for speeding up the generation of results. This area is still relatively new so it is likely that the classic algorithms have not yet been developed but several recent articles, e.g., (Pei et al. 2018) and (Kamiski and Szufel 2018), give an indication of how parallel computing can speed up these optimisation routines significantly.

A further area of research is designed to speed up optimisation by running initial tests on a low-fidelity model to obtain solutions that are likely to work well in the more complex simulation (and consequently the real system). In a recent article, (Xu et al. 2016), Xu and co-authors describe how the results of tests on low-fidelity models can be used to guide optimisation routines in the simulation models. The article refers to the use of digital twin technology and how optimisation can enhance this by not just mimicking the real system but suggesting better ways of running it into the future based on up-to-date input data. The process has some similarities to simheuristics (Juan et al. 2015), which are used to solve stochastic combinatorial optimisation problems. In a typical simheuristic algorithm, a metaheuristic is used to generate a set of good solutions for the deterministic version of the problem and these are then tested and refined using the simulation model.

\section{SUMMARY}

Our aim in this tutorial is to demonstrate the ease with which these sophisticated methods can be used in real simulations. The two key methods that we describe here and include in the Github repository are sequential methods, requiring constant communication between the simulation software and the optimisation algorithm. As discussed, this is sometimes not possible and we mentioned a possible alternative method (Monks and Currie 2018) that minimises these communications by going back to the two-stage models that were prevalent in the last century.

As discussed in the previous section, there are numerous variations on the original basic single-objective, single-solution problem, but understanding the algorithms for solving this original problem will provide an excellent base for understanding or indeed developing algorithms to solve the variations on the original problem.

In conclusion, if you need to guarantee the probability that you have selected the correct option for your system, use an indifference zone procedure such as $\mathrm{KN}++$. If instead you have a fixed computational budget, OCBA is a good option for maximising the probability that you choose the correct option in a set number of simulation replications.

Finally, the Github repository (https://github.com/TomMonks/ovs-tutorial) is there to be used - please do so! 


\section{Currie and Monks}

\section{ACKNOWLEDGMENTS}

Many thanks to the organisers of SW20 for giving us the opportunity to present this tutorial.

\section{REFERENCES}

Branke, J., S. E. Chick, and C. Schmidt. 2007. "Selecting a Selection Procedure". Management Science 53:1916-1932.

Chen, C.-H., D. He, M. Fu, and L. H. Lee. 2008. "Efficient Simulation Budget Allocation for Selecting an Optimal Subset". INFORMS Journal on Computing 20:579-595.

Chen, C.-H., and L. H. Lee. 2010, 07. Stochastic Simulation Optimization: An Optimal Computing Budget Allocation.

Chick, S. E., and K. Inoue. 2001. "New Two-Stage and Sequential Procedures for Selecting the Best Simulated System". Operations Research 49:732-743.

Fu, M. C. 2015. Handbook of Simulation Optimization. 1st ed. New York, USA: Springer.

Hong, L. J., and B. L. Nelson. 2009. "A brief introduction to optimization via simulation". In Proceedings of the 2009 Winter Simulation Conference, edited by M. D. Rossetti, R. R. Hill, B. Johansson, A. Dunkin, and R. G. Ingalls, 75-85. Piscataway, New Jersey: IEEE.

Hunter, S. R., E. A. Applegate, V. Arora, B. Chong, K. Cooper, O. Rincón-Guevara, and C. Vivas-Valencia. 2019, January. "An Introduction to Multiobjective Simulation Optimization". ACM Trans. Model. Comput. Simul. 29 (1): 7:1-7:36.

Juan, A. A., J. Faulin, S. E. Grasman, M. Rabe, and G. Figueira. 2015. "A review of simheuristics: Extending metaheuristics to deal with stochastic combinatorial optimization problems". Operations Research Perspectives 2:62-72.

Kamiski, B., and P. Szufel. 2018. "On parallel policies for ranking and selection problems". Journal of Applied Statistics 45 (9): 1690-1713.

Kim, S.-H., and B. L. Nelson. 2006. "Selecting the Best System". In Elsevier Handbooks in Operations Research and Management Science: Simulation, edited by S. G. Henderson and B. Nelson. Waltham, MA: Elsevier.

Koenig, L., and A. Law. 1985. "A Procedure for Selecting a Subset of Size m Containing the 1 Best of k Independent Normal Populations". Communications in Statistics - Simulation and Computation 14:719734.

Monks, T., and C. S. Currie. 2018. "Practical Considerations in Selecting the Best Set of Simulated Systems". In Proceedings of the 2018 Winter Simulation Conference, edited by M. Rabe, A. A. Juan, N. Mustafee, A. Skoogh, S. Jain, and B. Johansson, 2191-2200. Piscataway, New Jersey: IEEE.

Pei, L., S. Hunter, and B. Nelson. 2018. "A New Framework for Parallel Ranking \& Selection Using an Adaptive Standard". In Proceedings of the 2018 Winter Simulation Conference, edited by M. Rabe, A. A. Juan, N. Mustafee, A. Skoogh, S. Jain, and B. Johansson, 2201-2212. Piscataway, New Jersey: IEEE.

Xu, J., E. Huang, L. Hsieh, L. H. Lee, Q.-S. Jia, and C.-H. Chen. 2016. "Simulation optimization in the era of Industrial 4.0 and the Industrial Internet". Journal of Simulation 10 (4): 310-320.

\section{AUTHOR BIOGRAPHIES}

CHRISTINE CURRIE is Associate Professor of Operational Research in Mathematical Sciences at the University of Southampton, UK, where she also obtained her Ph.D. She is Editor-in-Chief for the Journal of Simulation. Christine was co-chair of the Simulation Special Interest Group in the UK Operational Research Society until September 2013. Her research interests include simulation optimisation, mathematical modelling of epidemics, optimal pricing and applications of simulation in health care. See http://www.southampton.ac.uk/maths/about/staff/ccurrie.page for more details. 
THOMAS MONKS is an Associate Professor of Health Data Science at the University of Exeter Medical School and The Institute of Data Science and AI, University of Exeter. He is also Turing Fellow at The Alan Turing Institute and Honorary researcher at the Clinical Operational Research Unit, UCL. Between 2014 and 2019 he led an applied healthcare Data Science team funded by the NIHR. He has worked as both a Software Engineer in the private sector and an Operational Research Analyst within the public sector. His speciality is simulation of healthcare systems of unscheduled and emergency care. He was co-chair of the UKs Simulation Workshop 2016 and 2018. His email is t.m.w.monks@exeter.ac.uk webpage is: https://www.turing.ac.uk/people/researchers/thomas-monks 